\title{
4-Floro-N-salisilidenanilinin Spektroskopik ve Doğrusal Olmayan Optik Özelliklerinin Belirlenmesi
}

\author{
Muhammet Hakk1 YILDIRIM ${ }^{*}$
}

\section{Öz}

Bir Schiff baz bileşiği olan 4-floro-N-salisilidenanilin bileşiğinin moleküler ve elektronik yapısı spektroskopik ve yoğunluk fonksiyoneli teorisi (YFT) metotları ile belirlendi. Seçilen teorik modelin doğruluğunu belirleyebilmek için YFT hesaplamalarından elde edilen moleküler bağ parametreleri ile deneysel tek kristal X-1şını kırınımı sonuçları karşılaştırıldı. Tatmin edici sonuçların ardından, FT-IR ve UV-Vis. spektrum hesaplamaları önceki teori seviyesi kullanılarak gerçekleştirildi. Tüm 72 normal titreşim modlarının bant atamaları, potansiyel enerji dağılım katkıları ile yapıldı. Keto-enol dönüşüm tautomerizmi, gaz ve çözücü ortamda teorik tarama hesaplamaları ile çalışıldı. Tarama hesaplamaları $\mathrm{O}-\mathrm{H} \cdots \mathrm{N}$ patikası boyunca gaz ve dört farklı çözücü ortamda gerçekleştirildi. Elde edilen sonuçlar hem katı hem de çözücü fazda enol yapının keto yapıdan daha baskın olduğunu gösterdi. Doğrusal olmayan optik özellik hesaplamaları bileşiğin iyi bir birinci dereceden hiper-kutuplanabilirli ğe sahip olduğunu göstermiştir.

Anahtar Kelimeler: Schiff baz, N-salisilidenanilin, Spektroskopi, YFT.

\section{Determining of Spectroscopic and Non-Linear Optical Properties of 4-Fluoro-N- salicylideneaniline}

\begin{abstract}
Molecular and electronic structure of a Schiff base compound 4-Fluoro-N-salicylideneaniline were determined by spectroscopic and density functional theory (DFT) methods. Molecular bond parameters which were obtained from DFT calculations were compared to experimental single crystal X-ray diffraction results to determine accuracy of the selected theoretical model. After the satisfactory results, FT-IR and UV-Vis. spectra calculations were performed by using the former level of theory. Band assignments of all 72 normal vibrational modes with potential energy distribution contributions were done. Keto-enol tautomerism was studied in gas and in solvent media by theoretical scan calculations. The scan calculations of proton transfer on the $\mathrm{O}-\mathrm{H} \cdots \mathrm{N}$ pathway in gas and four different media were performed. Obtained results show that the enol structure is more dominant than the keto structure both solid and solvent state. NLO calculations have been revealed that the compound has a good first order hyperpolarizability capacity.
\end{abstract}

Keywords: Schiff base, N-salicylideneaniline, Spectroscopy, DFT.

${ }^{1}$ Giresun Üniversitesi, Dereli Meslek Yüksekokulu, Giresun, Türkiye, hakki.yildirim@giresun.edu.tr

${ }^{1}$ https://orcid.org/0000-0001-6576-0252 


\section{Giriş}

İmin ( $>\mathrm{C}=\mathrm{N}-)$ grubu içeren bileşikler, 1864'deki Hugo Schiff ’in bu konudaki çalışmalarının (Schiff, 1864) ardından Schiff bazı olarak isimlendirilmiştir. Genel formülü RCH=NR' şeklinde olup, aldehit ve ketonların primer aminler (R'-NH2) ile reaksiyona girmeleri sonucunda oluşmaktadırlar (Tidwell, 2008). Schiff bazlarının elektronik yapısını ayrıntılı bir şekilde açığa çıkarmak için kristalografik, spektroskopik ve hesaplamalı yöntemleri bir arada bulunduran çalışmalar son yıllarda oldukça popülerdir (Albayrak Kaştaş vd., 2017; Eryılmaz vd., 2016; Kaştaş ve Kaştaş, 2019; Özdemir Tarı vd., 2016). Özellikle molekül içi hidrojen bağı nedeniyle farklı tautomerik yapılara sahip olabilen O-hidroksi Schiff bazları ilgi çekmektedir. Bunlar genellikle enol $(\mathrm{OH})$ ve keto $(\mathrm{NH})$ iki mümkün tautomerik formda gözlenirler. Bu iki olası tautomerik form, oksijen ve azot atomu arasında meydana gelen molekül içi hidrojen bağındaki hidrojen atomunun konumuna göre değişir. Bu bileşiklerde hidroksi oksijen atomundan imin azot atomuna proton transferiyle fotokromik ve termokromik özellik gösterdikleri bulunmuştur (Eugene Hadjoudis ve Mavridis, 2004). Fotokromizm, bir molekülün ışıkla renkli başka bir moleküle dönmesidir. Termokromizm de bir bileşiğin ısı etkisinde renkli başka bir moleküle dönüşmesidir. Her iki özellik de tersinirdir. Bazı termokromik ve fotokromik Schiff baz bileşiklerinden, molekülün geometrik yapısına bakılarak molekül düzlemsel ise termokromik, düzlemsel değilse fotokromik özellik gösterebileceği söylenebilir (E Hadjoudis vd., 1999). Fotokromik maddeler, optik veri depolama aletleri ve görüntü sistemlerinde kullanılabildikleri için son yıllarda bu tür maddeler üzerine çok sayıda çalışma yapılmaktadır (Cohen vd., 1964).

1999 yılında Burgess ve arkadaşları tarafından (Burgess vd., 1999) 4-Floro-N-salisilidenanilin (IUPAC ismi: 2-[(4-florofenil)iminometil] fenol, kısaca fsa) bileşiğinin moleküler bağ yapısı tek kristal X-ışını çalışmaları ile açığa çıkarılmıştır. Zaitsev ve arkadaşları fsa bileşiğinin çift dişli ligant özelliğini kullanarak alüminyum katkılı metal komplekslerini sentezlemişlerdir (Zaitsev vd., 2014). Sengar ve Narula fsa ve farklı ikincil ligantlar kullanarak 5 tane Eu(III) lüminesans özellikli kompleks sentezlemiş ve lüminesans özelliklerini açığa çıkarmışlardır (Sengar ve Narula, 2019). fsa'nın termokromik özelliğini kullanarak yeni ve daha verimli moleküller elde etmek için Mercier ve arkadaşları ko-kristal mühendisliği yaklaşımı ile 4 yeni kristal elde etmişlerdir (Mercier vd., 2016). Kaur ve arkadaşları $\mathrm{C}-\mathrm{H} \cdots \mathrm{F}$ hidrojen bağlarının paketleme üzerindeki etkilerini araştırmak için kullandıkları 20 bileşiğin içinde fsa bileşiğinin de moleküler paketlenmesini açığa çıkarmışlar ve düşük sıcaklık tek kristal x-1şını kırınım verilerini yayınlamışlardır. Hem Schiff bazlarının hem de fsa bileşiğinin ilgi çekici özelliklerine rağmen, bildiğimiz kadarıyla fsa bileşiğinin kendi başına detaylı spektroskopik ve teorik çalışmaları yapılmamıştır. Bu çalışmada bileşiğin hesaplamalı yöntemlerle desteklenen spektroskopik analizleri ile doğrusal olmayan optik özellikleri için teorik veriler de ilk defa olarak verilmektedir. 


\section{Materyal ve Metot}

Bileşiğin katı haldeki moleküler yapısı Burgess ve ark. ile Kaur ve ark. tarafından yapılan tek kristal X-ışını çalışmalarından alınmıştır (Burgess vd., 1999; Kaur vd., 2016). Bileşiğin spektroskopik verileri Spectrabase web sitesinden alınmıştır (Bio-Rad Laboratories, 2019). FT-IR spektrumu Bruker Tensor 27 FT-IR spektrometresi kullanılarak, KBR disk yöntemi ile ve UV-Vis. spektrumu metanol çözücüsü içinde 200-500 nm aralığında elde edilmiştir. ${ }^{1} \mathrm{H}$ ve ${ }^{13} \mathrm{C}$ NMR spektrumları sırasıyla Varian A-60 ve CFT-20 spektrometreleri ile, chloroform-d çözücüsünde toplanmıştır.

$\mathrm{Bu}$ çalışmadaki tüm hesaplamalar, Gaussian 09W paket programında (Frisch vd., 2009), yoğunluk fonksiyoneli teorisinde B3LYP (Becke, 1993) hibrit fonksiyoneli ile 6-311++G(d,p) baz seti kullanılarak gerçekleştirilmiştir. İlaveten bileşiğin optimizasyonu için Minnesota 06 fonksiyonellerinden (Zhao ve Truhlar, 2008) M06-2X fonksiyoneli ile Karlsruhe baz setlerinden def2TZVP (Weigend ve Ahlrichs, 2005) baz seti karşılaştırma amaçlı kullanılmıştır. Teorik hesaplamaların görselleştirilmesinde GaussView 5 programı (Dennington vd., 2009) kullanılmıştır.

\section{Bulgular ve Tartışma}

\subsection{Moleküler Yapının Hesaplamalı Yöntemle Modellenmesi}

Kaur ve arkadaşları tarafından $100^{\circ} \mathrm{K}$ sıcaklıkta gerçekleştirilen tek kristal X-1şını kırınımı deneyindeki verilerden (Kaur vd., 2016) yararlanılarak elde edilen atom isimlendirilmiş \%50 1sısal olasılık elipsleri ve B3LYP ile hesaplanmış molekül yapıları Şekil 1’de verilmiştir.
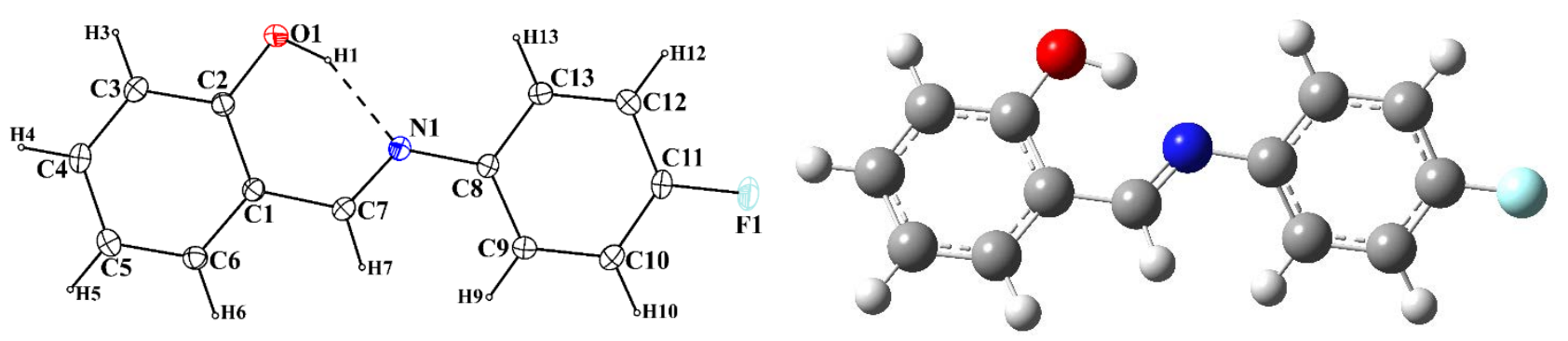

Şekil 1. fsa bileşiğinin \%50 termal olasılıklı elipsoitlerle temsil edilen (sol) ve hesaplanan (sağ) moleküler yapilar1

Bir molekülün geometrik parametreleri elektron yoğunlukları ile doğrudan ilgili olduğundan seçilen hesaplamalı yöntemin geometrik parametreleri belirlemedeki başarısı diğer elektronik özelliklerinin modellenmesi için bir kıstas olarak belirlenebilir. Bu amaçla bileşiğin optimize 
moleküler yapısı hem B3LYP/6-311++(d,p) hem de M062X/def2TZVP yöntemi ile hesaplandı. Seçili bağ parametreleri için elde edilen deneysel ve hesaplamalı değerler Tablo 1.'de verildi.

Tablo 1. Bileşiğin seçili bağ geometrilerinin deneysel ve hesaplanan değerleri

\begin{tabular}{|c|c|c|c|c|}
\hline Bağ Uzunluğu $(\AA)$ & $\mathrm{XRD}^{\mathrm{a}}$ & $\mathrm{XRD}^{\mathrm{b}}$ & B3LYP & M062X \\
\hline N1-C7 & 1,273 & 1,290 & 1,289 & 1,277 \\
\hline N1-C8 & 1,419 & 1,417 & 1,408 & 1,408 \\
\hline O1-C2 & 1,353 & 1,355 & 1,341 & 1,335 \\
\hline O1-H1 & 0,943 & 0,894 & 0,992 & 0,986 \\
\hline C1-C7 & 1,443 & 1,451 & 1,449 & 1,452 \\
\hline C7-H7 & 0,930 & 0,950 & 1,096 & 1,096 \\
\hline C8-C13 & 1,378 & 1,400 & 1,402 & 1,394 \\
\hline F1-C11 & 1,362 & 1,363 & 1,356 & 1,341 \\
\hline \multicolumn{5}{|l|}{ Bağ Açısı ( $\left.{ }^{\circ}\right)$} \\
\hline N1-C7-C1 & 122,54 & 121,15 & 122,55 & 122,90 \\
\hline N1-C7-H7 & 118,73 & 119,41 & 121,11 & 120,85 \\
\hline C8-N1-C7 & 123,08 & 122,07 & 121,27 & 120,20 \\
\hline C9-C8-N1 & 124,99 & 125,39 & 123,09 & 122,61 \\
\hline C13-C8-N1 & 116,03 & 115,45 & 117,93 & 118,06 \\
\hline C1-C2-O1 & 121,56 & 121,64 & 121,90 & 122,13 \\
\hline C1-C7-H7 & 118,74 & 119,45 & 116,34 & 116,25 \\
\hline C2-C1-C7 & 121,56 & 121,85 & 121,45 & 121,51 \\
\hline C3-C2-O1 & 118,62 & 118,71 & 118,62 & 118,52 \\
\hline C10-C11-F1 & 118,50 & 118,42 & 118,91 & 118,96 \\
\hline C12-C11-F1 & 118,79 & 118,56 & 118,94 & 118,96 \\
\hline C13-С8-C9 & 118,98 & 119,16 & 118,95 & 119,30 \\
\hline \multicolumn{5}{|l|}{ Burulma Açısı $\left(^{\circ}\right)$} \\
\hline C2-C1-C7-H7 & 175,4 & 174,4 & 179,9 & 179,5 \\
\hline C2-C1-C7-N1 & $-4,6$ & $-5,6$ & 0,7 & 0,4 \\
\hline C6-C1-C7-H7 & $-5,0$ & $-6,1$ & $-0,1$ & $-0,3$ \\
\hline C6-C1-C7-N1 & 175,0 & 174,0 & $-179,2$ & $-179,4$ \\
\hline C7-N1-C8-C13 & $-173,8$ & $-173,6$ & $-145,3$ & $-143,1$ \\
\hline C7-N1-C8-C9 & 6,1 & 6,8 & 37,1 & 39,0 \\
\hline C8-N1-C7-C1 & $-179,1$ & $-178,9$ & $-177,2$ & $-178,0$ \\
\hline C8-N1-C7-H7 & 0,9 & 1,1 & 3,7 & 2,9 \\
\hline H1-O1-C2-C1 & 1,9 & 2,6 & $-0,3$ & $-0,3$ \\
\hline
\end{tabular}

Ölçülen ve hesaplanan değerler arasındaki sapmanın miktarını belirlemenin yollarından birisi de kare ortalama karekök sapmasını (RMSE) bulmaktır. Düşük sıcaklıkta verilerin toplandığı dolayısıyla bağ parametrelerinin daha güvenilir olduğu Kaur ve arkadaşlarının x-1şını çalışması referans alınarak her iki yöntem için de RMSE hesaplamaları yapıldı. Hesaplamalar hidrojenlerinki de dahil olmak üzere tüm bağ parametreleri kullanılarak, uzunluk, açı ve burulma olmak üzere üç grupta yapıldı. Bağ uzunluklarında B3LYP yönteminde sapma 0,078 Å iken M06-2X için 0,077 $\AA$ 
olarak bulunmuştur. B3LYP yöntemi bağ açılarında ve burulmalarında sırasıyla $1,00^{\circ}$ ve $5,8^{\circ}$ sapmaya sahipken aynı değerler diğer yöntem için $1,08^{\circ}$ ve $6,1^{\circ}$ olarak hesaplanmıştır. Hesaplamaların izole tek bir molekül için gerçekleştirilmesi yüzünden moleküller arası etkileşimlerin dikkate alınmadığı hesaba katılırsa sonuçlardan her iki modelin de yeterli doğrulukta (İng.: accuracy) olduğu söylenebilir. Başlıktaki bileşiğin B3LYP optimizasyonu için işlemci zamanı (Ing.: cpu time) 1 saat 29 dakika 48 saniye iken aynı bilgisayarda M06-2X yöntemi 4 saat 52 dakika 26 saniye olarak gerçekleşti. Hesaplamalı yöntemlerde model seçerken dikkat edilen bir diğer parametre olan maliyet de dikkate alınarak bileşiğin diğer hesaplamaları sadece B3LYP yöntemi ile yapıldı.

Schiff bazlarında $\mathrm{C}-\mathrm{N}$ ve $\mathrm{C}-\mathrm{O}$ bağ uzunlukları gösterge bağ uzunlukları olarak adlandırılır ve bileşiğin tautomerisinin belirlenmesinde kullanılırlar (Karabiyik vd., 2009). Azot atomunun komşu karbon atomları ile yaptığı N1—C7 çift ve N1—C8 tek bağları deneysel olarak sırasıyla 1.273 (2) ve 1.419 (2) Å bulunmuş iken optimize yapıda 1.289 ve $1.408 \AA$ olarak hesaplanmıştır. Hidroksi grubunun benzen halkasıyla yaptığı bağ, deneysel olarak 1.362 (2) Å uzunluğunda tipik bir tek bağ iken 1.341 Å olarak hesaplanmıştır. Bu bağlar literatürdeki benzer yapılarla (Demircioğlu vd., 2018; Özdemir Tarı vd., 2018) uyumlu ve bileşiğin katı fazda enol yapıda olduğunu doğrulamaktadır.

Poliaromatik bileşiklerde, aromatik halka düzlemlerinin arasındaki açı molekülün düzlemselliği hakkında bilgi verir. Bileşik kristal halde neredeyse düzlemsel $\left(2,16^{\circ}\right)$ olmasına rağmen gaz faz için hesaplanan yapıda $\left(38,30^{\circ}\right)$ olarak bulunmuştur. Bu durumun gaz fazı hesaplamalarında paketlenme etkilerinin dikkate alınamamasından kaynaklandığı belirtilmiştir (Özek Yıldırım vd., 2016). Bu nedenle bileşiğin katı halde termokromik özellik gösterebileceği, sıv1 fazda ise fotokromik özellik gösterebileceği söylenebilir.

\subsection{Keto-enol Tautomerizm Dönüşüm Bariyeri Taraması}

Schiff bazlarında, dışardan verilen termal ya da ışın enerjisi ile keto-enol dönüşümü yapılabilir ve bu sayede bileşiğin fotokromik yada termokromik özellik göstermesi sağlanabilir (Ünver vd., 2008). Bu dönüşümü gerçekleştirmek için gerekli olan enerji miktarı oksijen atomundan azot atomuna doğru göç ettirilen protonun molekülün toplam enerjisindeki değişimine bakılarak bulunur ve potansiyel enerji bariyeri olarak adlandırılır (Tezer ve Karakus, 2009). Bu bariyerin yüksekliği çözücüye yüksek oranda bağlı olduğu için, molekülün potansiyel enerji bariyeri tarama yöntemi ile gaz, metoksi, benzen, kloroform ve DMSO çözücüleri içinde hesaplandı. Tarama yönteminde, oksijen atomuna bağlı olan proton, 20 adım boyunca, her adımda $0.05 \AA$ azot atomuna doğru yaklaştırılarak optimize edildi. Her optimizasyonda sadece oksijen-hidrojen bağı sabit tutuldu, diğer tüm parametreler optimize edildi ve toplam enerji değerleri en düşük enerjili olan (DMSO içinde $1 \AA$ 
$d(\mathrm{O}-\mathrm{H}))$ şekillenime göre göreli toplam enerjiler hesaplandı. Elde edilen tarama eğrileri Şekil 2.'de verildi.

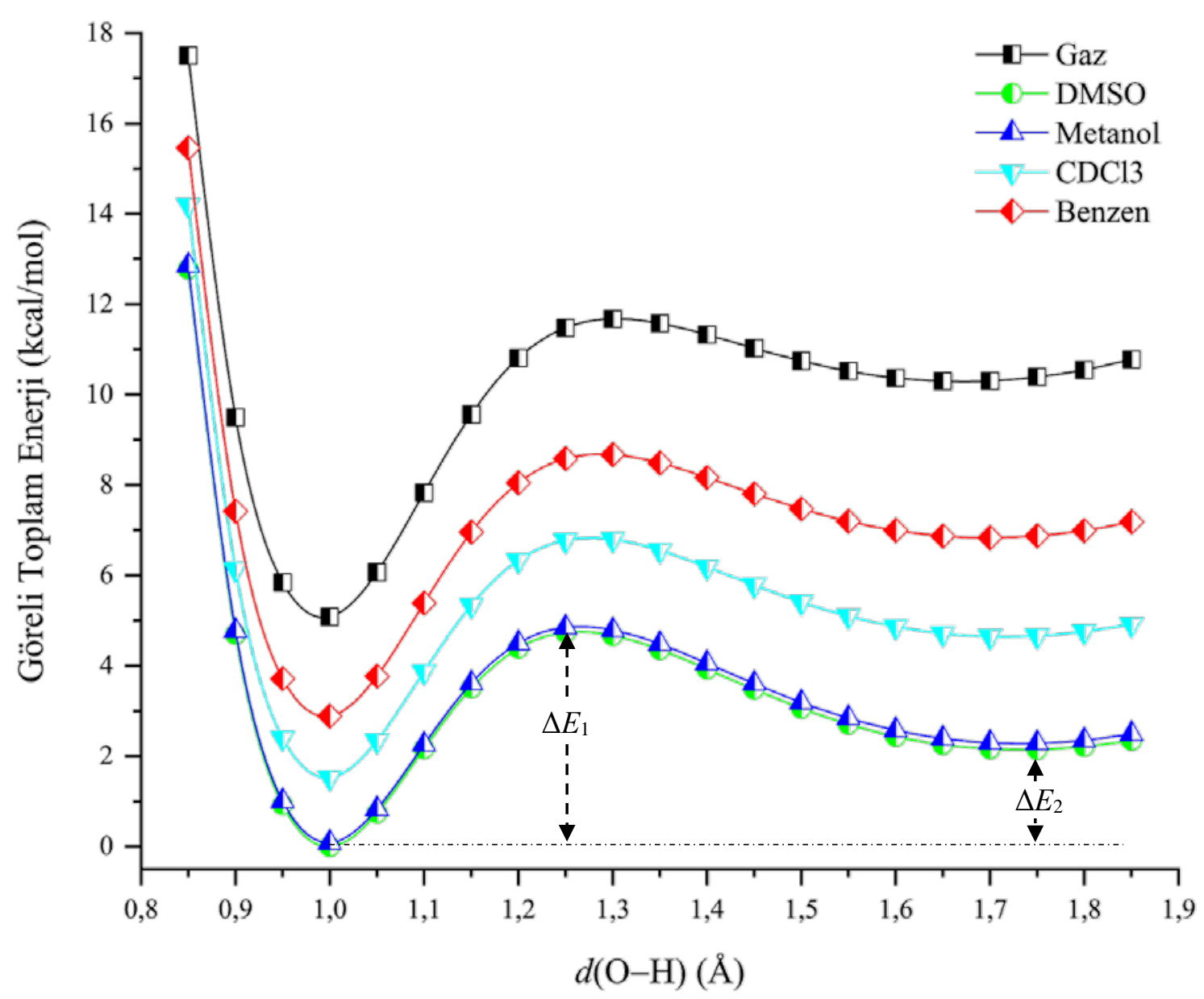

Şekil 2. fsa molekülünün proton transfer sürecindeki tek boyutlu toplam enerji değişimi

Yapılan hesaplamalardan molekülün en düşük enerjili formunun, DMSO çözeltisi içinde O-H bağ uzunluğunun $1 \AA$ olduğu durum olarak belirlendi. Proton transferi için gerekli enerji miktarı ya da diğer adıyla keto-enol enerji bariyeri $\left(\Delta E_{1}\right)$; DMSO, metanol, kloroform, benzen ve gaz için sırasıyla, 4,7, 4,8, 5,3, 5,8 ve 6,6 kcal/mol olarak elde edilmiştir. Molekülün keto-enol durumları arasındaki enerji farkı $\left(\Delta E_{2}\right)$ aynı ortamlar için sırasıyla 2,1, 2,2, 3,1, 3,9 ve 5,2 kcal/mol olarak bulunmuştur. Molekülün farklı ortamlardaki toplam enerjilerinde, keto-enol bariyerinde ve keto-enol enerji farkındaki artışın ortamın elektrostatik potansiyel değeri (eps) değeri (DMSO: $\varepsilon=46.82$ Metanol: $\varepsilon=32.61$ Kloroform: $\varepsilon=4.71$ Benzen: $\varepsilon=2.27$ ) ile ters orant1lı olduğu ve ortamın polaritesi arttıkça enerji bariyerinin azaldığı görülmüştür. Bu sonuç Dudek ve Dudek (Dudek ve Dudek, 1966) tarafından yapılan Schiff bazlarında keto-enol dengesinin deneysel NMR ile incelenmesi çalışmasında elde edilen polar çözücülerde enol formun daha baskın olması sonucu ile uyumludur. 


\subsection{FT-IR Spektroskopisi ile Titreşim Analizleri}

Gaussian 09 programında titreşimsel frekanslar, enerjinin kartezyen nükleer koordinatlara göre ikinci türevinden hesaplanmakta ve çekirdek konumlarına doğrudan bağlıdır (Foresman ve Frisch, 1996). Bu nedenle optimize olmuş kararlı bir yapıda hesaplanan frekans değerleri moleküler modellemede en önemli adımdır. Bu yüzden ölçülen ve hesaplanan değerler arasındaki bu uyum modellemenin doğruluğunu göstermektedir.

Hesaplanan IR spektrumlarında sistematik hataları elimine etmek için Andersson ve Uvdal tarafından önerilen 0,9679 (Andersson ve Uvdal, 2005) ölçeklendirme çarpanı kullanılmıştır. Bileşiğin $\mathrm{KBr}$ pellet tekniği ile çekilen katı fazdaki Fourier dönüşümlü kırmızı-altı spektrumu ile hesaplamalı yöntemden elde edilen kırmızı-altı spektrumu karşılaştırmalı olarak Şekil 3.’de verilmiştir.

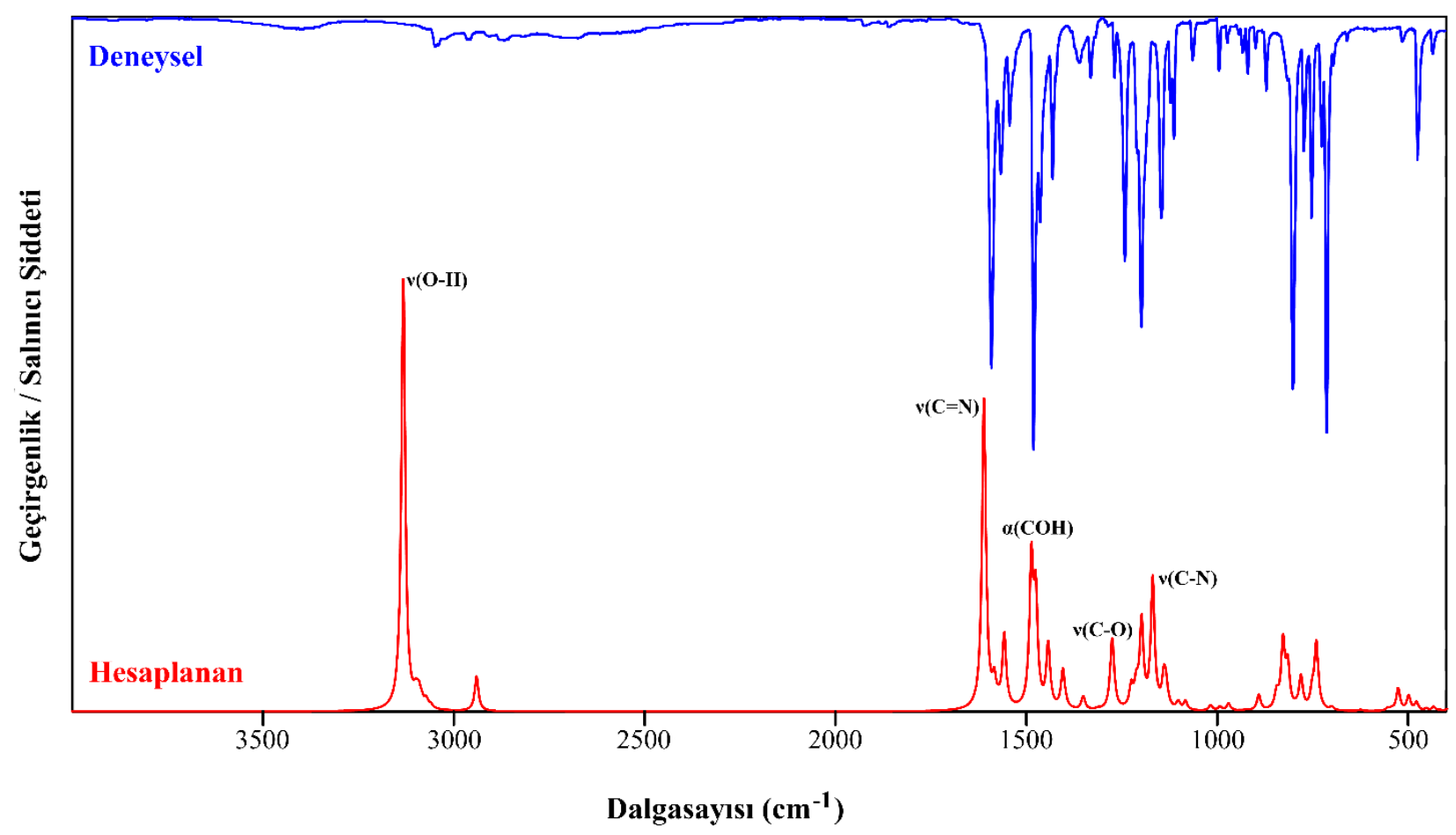

Şekil 3. Bileşiğin deneysel FT-IR spektrumu (mavi-üstte) ve hesaplanan IR spektrumunun (kırmızı-altta) karşılaştırılması

C1 nokta simetrisine sahip fsa bileşiğinin toplam 26 atomdan oluştuğu için 72 normal titreşim koordinatına sahiptir. Bileşiğin deneysel IR spektrumundan ayırt edilebilen titreşim değerleri, tüm koordinatlarına ait hesaplanan titreşim değerleri ile bu titreşimlerin bileşenleri potansiyel enerji dağılımı analizi yardımı belirlenmiş ve Tablo 2.'de verilmiştir. 
Tablo 2. Bileşiğin hesaplanan IR titreşimleri ve bant atamalarının potansiyel enerji dağılımları

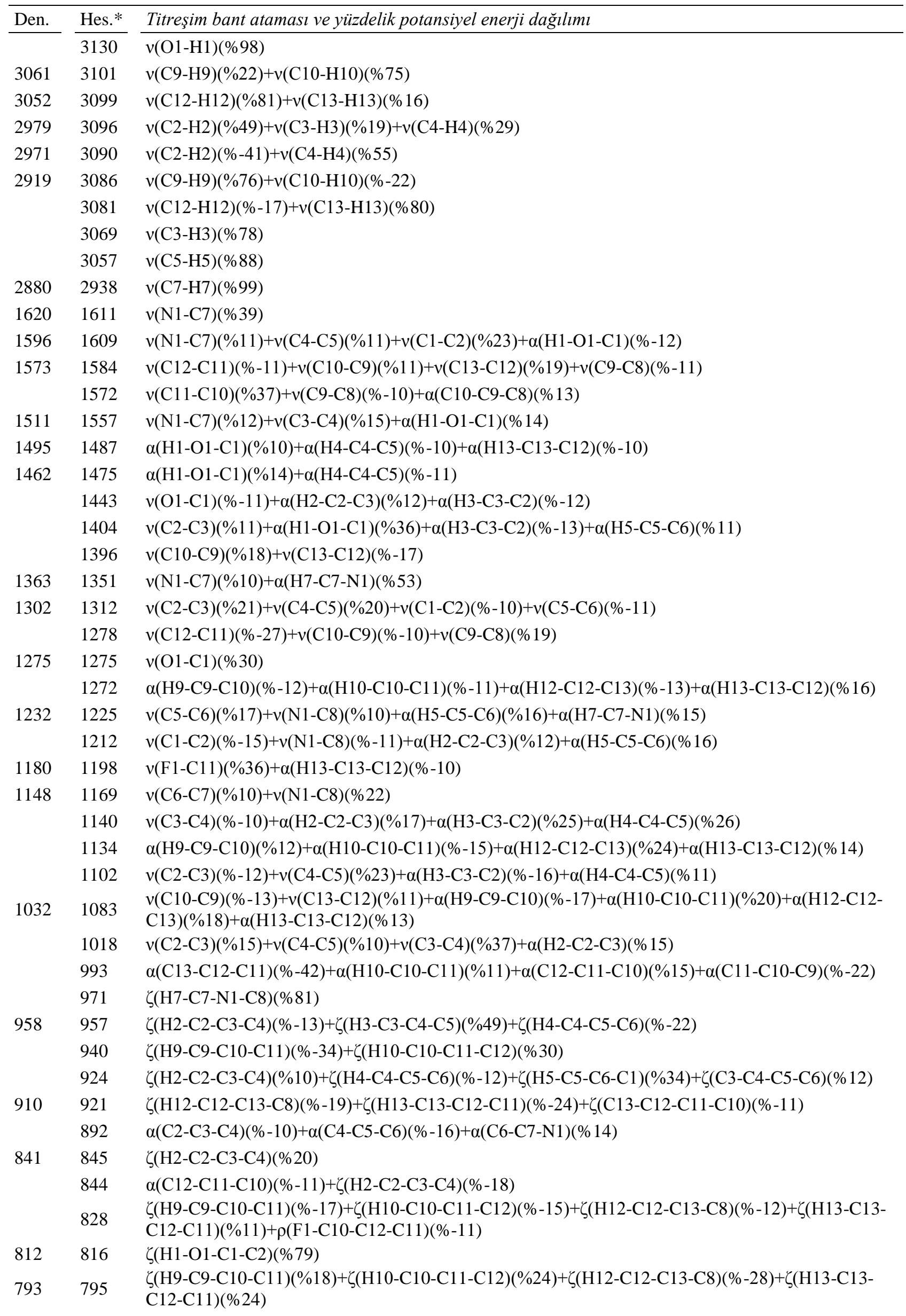




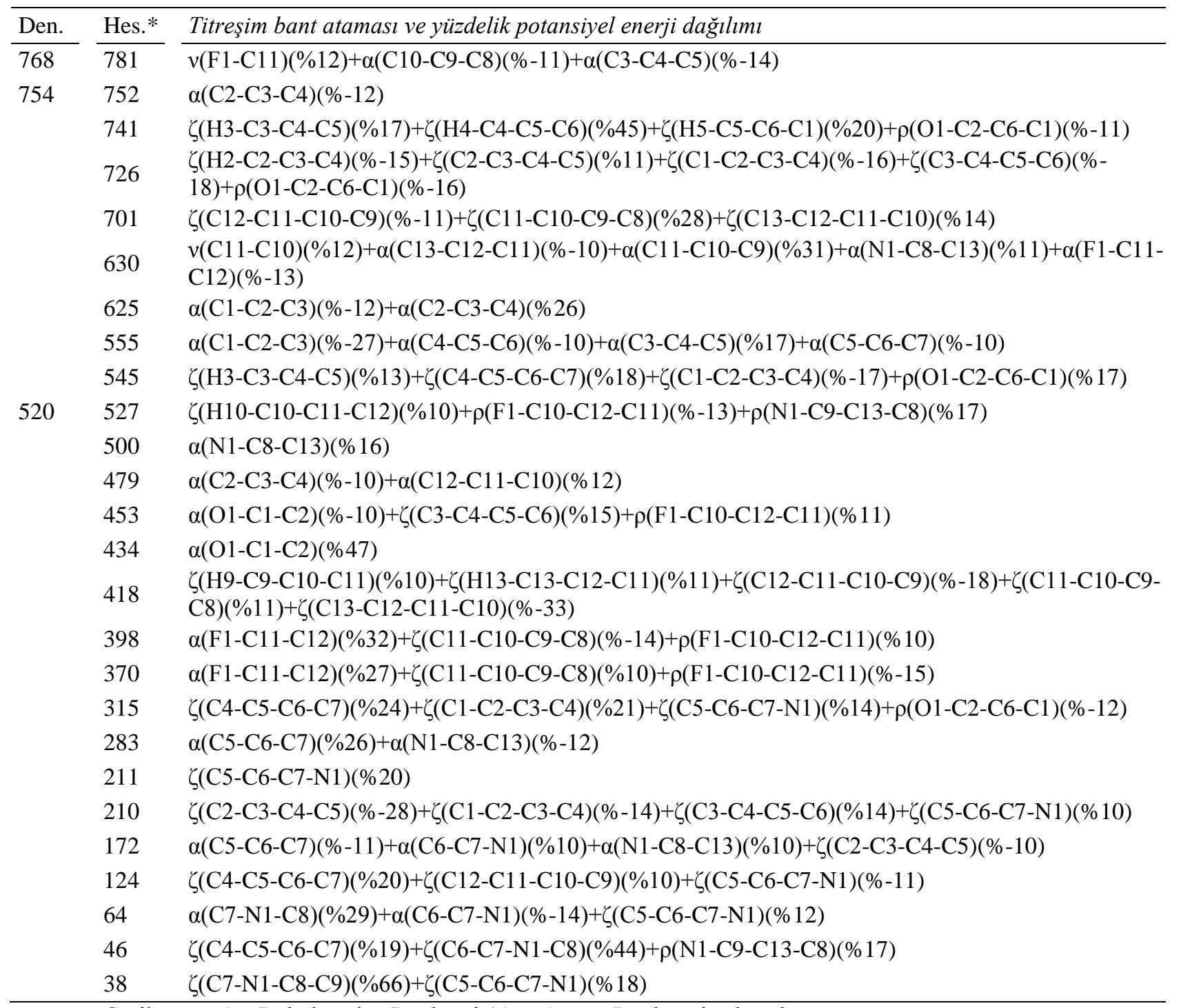

v: Gerilme, $\alpha$ : Açı Bükülmesi, $\tau$ :Düzlem içi burulma, $\rho:$ Düzlem dışı burulma

*0,9679 (Andersson ve Uvdal, 2005)

Gösterge titreşimlere bakıldığında, hidroksi grubunun gerilme titreşimi molekül içi güçlü hidrojen bağı nedeniyle deneysel spektrumda yayvan bir bant şeklinde iken $3081 \mathrm{~cm}^{-1}$ olarak hesaplanmıştır. Bir diğer gösterge titreşim olan $\mathrm{C}=\mathrm{N}$ gerilmesi $1611 \mathrm{~cm}^{-1}$, de olarak hesaplanmış ve deneysel spektrumda $1620 \mathrm{~cm}^{-1}$ 'de gözlenmiştir. Diğer gözlenen ve hesaplanan değerler tablodan görüleceği üzere uyum içindedir.

\subsection{UV Spektroskopisi ile Elektronik Geçiş Analizi}

Schiff bazlarında UV spektrumunda $400 \mathrm{~nm}$ üzerinde görülen maksimum bileşiğin keto formundan kaynaklanır ve keto-enol dengesi hakkında bilgi verir (Ünver vd., 2001). Bileşiğin metanol çözücüsü içinde çekilmiş UV spektrumu ve zamana bağlı YFT yöntemi ile elde edilmiş UV spektrumları Şekil 4.'de verildi. Şekilden görüldüğü üzere $400 \mathrm{~nm}$ üzerinde geçişin bulunmaması 
bileşiğin sıvı formda da enol formunu benimsediğini göstermektedir. Potansiyel enerji taramasında da belirtiği üzere polar çözücülerde keto-enol bariyeri daha düşük olmasına rağmen bileşiğin bu formda bulunması takı etkilerinden kaynaklanmaktadır.

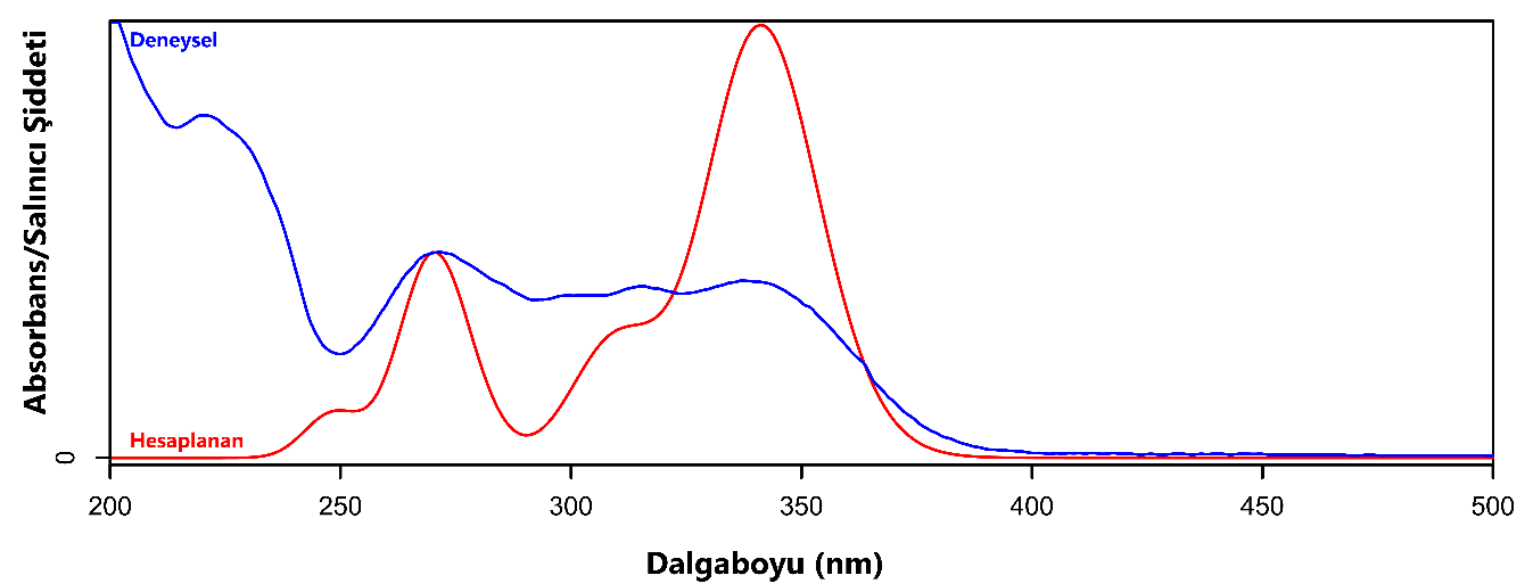

Şekil 4. Bileşiğin metanol çözücüsü içindeki gözlenen ve hesaplanan UV spektrumları

UV spektrumundaki maksimumların hangi moleküler orbitaller arasındaki geçişten kaynaklandığını belirlemek için majör katkılar Gaussum programı (O’boyle vd., 2008) yardımı ile hesaplanarak Tablo 3.’deki gibi verildi.

Tablo 3. Gözlenen ve hesaplanan elektronik geçişlerin türleri ve bileşenleri

\begin{tabular}{|c|c|c|c|c|}
\hline Den.(nm) & Hes. (nm) & Geçiş & $\begin{array}{l}\text { Salınıcı } \\
\text { Şiddeti }\end{array}$ & Majör Katkılar \\
\hline 337 & 341 & $\mathrm{n} \rightarrow \pi^{*}$ & 0.501 & $\mathrm{H} \rightarrow \mathrm{L}(96 \%)$ \\
\hline 315 & 310 & $\mathrm{n} \rightarrow \pi^{*}$ & 0.141 & $\mathrm{H}-1 \rightarrow \mathrm{L}(91 \%)$ \\
\hline \multirow[t]{2}{*}{271} & 272 & $\pi \rightarrow \pi^{*}$ & 0.124 & $\mathrm{H}-3 \rightarrow \mathrm{L}(12 \%), \mathrm{H}-2 \rightarrow \mathrm{L}(51 \%), \mathrm{H} \rightarrow \mathrm{L}+1(20 \%)$ \\
\hline & 269 & & 0.118 & $\mathrm{H}-4 \rightarrow \mathrm{L}(12 \%), \mathrm{H}-3 \rightarrow \mathrm{L}(37 \%), \mathrm{H}-2 \rightarrow \mathrm{L}(19 \%), \mathrm{H} \rightarrow \mathrm{L}+1(22 \%)$ \\
\hline
\end{tabular}

H: Dolu orbitallerin en yüksek enerjilisi, L: Boş orbitallerin en düşük enerjilisi

Schiff baz köprüsünde bulunan azot atomunun eşleşmemiş elektronlarının uyarılmaları ile gerçekleşen $n \rightarrow \pi^{*}$ geçişleri bileşiğin UV spektrumundaki enol formunu tercih ettiğini gösterir. Sengar ve Nagula tarafından fsa bileşiğinin metal kompleksi çalışmalarında da benzer durum rapor edilmiş (Sengar ve Narula, 2019) ve bileşiğin komplekslerinde $300 \mathrm{~nm}$ üzerindeki geçişlerin yok olması ligant-metal bağının kurulmasına yorulmuştur.

\subsection{Doğrusal Olmayan Optik Özellikler}

Doğrusal olmayan optik (NLO) özellik maddenin bir özelliği olup, doğrusal olmayan ortamlarda: kırılma indisi gelen 1şı̆̆ın şiddetine bağlı, gelen ışığın frekansı maddeden çıtığında 
değişebilir ve çıkan 1şık başka bir 1şık ile kontrol edilebilir (Sarı, 2020). Bu nedenle doğrusal olmayan optik özellik gösteren madde tasarımı ve üretimi optoelektronik alanındaki en popüler konulardandır. En temel doğrusal olmayan optik özellik gösteren molekül tasarımı, donör ve akseptör gruplarının bir $\pi$ bağı ile bağlanmasıdır (Bosshard vd., 2000). Schiff bazlarında var olan azometin köprüsü ve yüksek delokalize elektronlara sahip aromatik halkalar bu bileşiklerin NLO özelliklerinin yüksek olmasını sağlamakta ve araştırmacıların ilgisini çekmektedir. Molekülün taban durum dipol momentleri $\mu$, kutuplanabilirlik parametreleri $\alpha$ ve birinci mertebe aşırı kutuplanabilirlik (hyperpolarizability) parametreleri $\beta$, Thanthiriwatte ve Silva'nın önermiş olduğu şekilde (Thanthiriwatte ve Nalin de Silva, 2002) hesaplandı ve Tablo 4.'de verildi.

Tablo 4. Molekülün hesaplanan optik özellikleri

\begin{tabular}{|c|c|c|c|c|c|}
\hline \multicolumn{2}{|c|}{ Dipol Moment (Debye) } & \multicolumn{2}{|c|}{ Kutuplanabilirlik $\left(\AA^{3}\right)$} & \multicolumn{2}{|c|}{ Aşırı Kutuplanabilirlik (a.u.) } \\
\hline$\mu_{\mathrm{x}}$ & $-1,2$ & $\alpha_{\mathrm{xx}}$ & 303,2 & $\beta_{\mathrm{xxx}}$ & $-727,2$ \\
\hline$\mu_{\mathrm{y}}$ & $-2,0$ & $\alpha_{\mathrm{xy}}$ & 7,3 & $\beta_{\text {xxy }}$ & $-240,5$ \\
\hline$\mu_{\mathrm{z}}$ & 0,4 & $\alpha_{\mathrm{yy}}$ & 158,3 & $\beta_{\text {xyy }}$ & $-46,6$ \\
\hline \multirow[t]{8}{*}{$\mu_{\text {ort }}$} & 2,4 & $\alpha_{\mathrm{xz}}$ & 0,5 & $\beta_{\text {yуy }}$ & $-126,1$ \\
\hline & & $\alpha_{\mathrm{yz}}$ & 3,0 & $\beta_{\mathrm{xxz}}$ & 100,3 \\
\hline & & $\alpha_{\mathrm{zz}}$ & 93,9 & $\beta_{\mathrm{xyz}}$ & 21,6 \\
\hline & & $\Delta \alpha$ & 15,5 & $\beta_{\mathrm{yyz}}$ & 21,1 \\
\hline & & & & $\beta_{\mathrm{xzz}}$ & $-64,0$ \\
\hline & & & & $\beta_{\mathrm{yzz}}$ & $-40,5$ \\
\hline & & & & $\beta_{\mathrm{zzz}}$ & 33,6 \\
\hline & & & & $\beta_{\text {top }}\left(\mathrm{cm}^{5} / \mathrm{esu}\right)$ & $8,16 \times 10^{-30}$ \\
\hline
\end{tabular}

Donaldson ve Tang'ın yaptığı çalışmada (Donaldson ve Tang, 1984), üre kristallerinin KDP kristali yerine kullanılabildiğini deneysel olarak ortaya koyarak hem üretimi basit hem de ucuz olan organik materyallerden NLO materyal üretilebileceğini göstermiş oldu. Bu dönemden sonra yapılan NLO çalışmalarında üre bir karşılaştırma maddesi olarak kullanıldı. Ledox ve Zyss (Ledoux ve Zyss, 1982) ürenin aşırı kutuplanabilirlik değerini $0.45 \times 10^{-30} \mathrm{~cm}^{5} / \mathrm{esu}$ olarak belirledi. Bu verilerden fsa bileşiğinin ürenin 18 katı bir aşırı kutuplanabilirlik gösterdiği söylenebilir.

\section{Sonuçlar ve Öneriler}

YFT/B3LYP/6-311G++(d,p) ve YFT/M06-2x/def2TZVP yöntemleri ile yapılan moleküler geometri optimizasyonunda B3LYP yönteminin yeterli doğrulukta olduğu görüldü. Tarama hesaplamalarında, keto-enol dönüşüm bariyerinin yüksekliği DMSO gibi yüksek polariteli çözeltide $4,7 \mathrm{kcal} / \mathrm{mol}$ ve gaz fazında $6,6 \mathrm{kcal} / \mathrm{mol}$ olarak elde edildi. Bileşiğin 72 normal titreşim koordinatının 
tümü potansiyel enerji dağılımı yöntemi ile bileşenlerine ayrılarak atamaları yapıldı ve deneysel verilerle karşılaştırıldı. Bileşiğin kristal halde iken bulunduğu enol formunun, metanol içinde çekilen UV spektrumu incelenerek, çözelti içinde de baskın olduğu görüldü. Bileşiğin hesaplanan NLO değerleri üre ile kıyaslandığında iyi bir NLO materyal olma kapasitesine sahip olduğu söylenebilir. Bu çalışmalar aynı zamanda sonraki çalışmalar için de yardımcı olacaktır.

\section{Kaynaklar}

Albayrak Kaştaş, Ç., Kaştaş, G., Güder, A., Gür, M., Muğlu, H. ve Büyükgüngör, O., (2017). Investigation of two o-hydroxy Schiff bases in terms of prototropy and radical scavenging activity. Journal of Molecular Structure, 1130, 623-632.

Andersson, M. P. ve Uvdal, P., (2005). New scale factors for harmonic vibrational frequencies using the B3LYP density functional method with the triple- $\zeta$ basis Set $6-311+G(d, p)$. The journal of physical chemistry. A, 109(12), 2937-2941.

Becke, A. D., (1993). Density-functional thermochemistry. III. The role of exact exchange. The Journal of Chemical Physics, 98(7), 5648.

Bio-Rad Laboratories, I. S., (2019). Bio-Rad Laboratories, Inc. SpectraBase; SpectraBase Compound ID=5Y3IKqtn0oh. http://spectrabase.com/compound/5Y3IKqtn0oh adresinden erişildi

Bosshard, C., Bösch, M., Liakatas, I., Jäger M., ve Günter, P., (2000). Second-Order Nonlinear Optical Organic Materials: Recent Developments, Nonlinear Optical Effects and Materials, Peter Günter (Ed.), (ss. 163299). New York, NY: Springer.

Burgess, J., Fawcett, J., Russell, D. R., Gilani, S. R. ve Palma, V., (1999). Four N -(2hydroxybenzylidene)aniline derivatives. Acta Crystallographica Section C Crystal Structure Communications, 55(10), 1707-1710.

Cohen, M. D., Schmidt, G. M. J. ve Flavian, S., (1964). Topochemistry. Part VI. Experiments on Photochromy and Thermochromy of Crystalline Anils of Salicylaldehydes. Journal of the Chemical Society, 20412051.

Demircioğlu, Z., Kaştaş, Ç. A. ve Büyükgüngör, O., (2018). XRD, FT-IR and UV characterization, hirshfeld surface analysis and local-global chemical descriptor studies of (E)-2-((3-fluorophenylimino)methyl)3-methoxyphenol (1) and (E)-2-((2-fluorophenylimino)methyl)-3-methoxyphenol (2). Journal of Molecular Structure, 1166, 131-146.

Dennington, R., Keith, T. ve Millam, J., (2009). GaussView, Version 5, Semichem Inc., Shawnee Mission, KS.

Donaldson, W. R. ve Tang, C. L., (1984). Urea optical parametric oscillator. Applied Physics Letters, 44(1), 25-27.

Dudek, G. O. ve Dudek, E. P., (1966). Spectroscopic Studies of Keto-Enol Equilibria. IX. N 15 -Substituted Anilides 1,2. Journal of the American Chemical Society, 88(11), 2407-2412.

Eryılmaz, S., Gül, M., İnkaya, E., İdil, Ö. ve Özdemir, N., (2016). Synthesis, crystal structure analysis, spectral characterization, quantum chemical calculations, antioxidant and antimicrobial activity of 3-(4chlorophenyl)-3a,4,7,7a-tetrahydro-4,7-methanobenzo[d]isoxazole. Journal of Molecular Structure, 1122, 219-233.

Foresman, J. B. ve Frisch, A., (1996). Exploring Chemistry with Electronic Structure Methods (Second Ed.). Wallingford, CT, Gaussian Inc..

Frisch, M. J., Trucks, G. W., Schlegel, H. B., Scuseria, G. E., Robb, M. A., Cheeseman, J. R., Scalmani, G., Barone, V., Petersson, G. A., Nakatsuji, H., Li, X., Caricato, M., Marenich, A. V., Bloino, J., Janesko, B. G., Gomperts, R., Mennucci, B., Hratchian, H. P., Ortiz, J. V., Izmaylov, A. F., Sonnenberg, J. L., Williams-Young, D., Ding, F., Lipparini, F., Egidi, F., Goings, J., Peng, B., Petrone, A., Henderson, T., Ranasinghe, D., Zakrzewski, V. G., Gao, J., Rega, N., Zheng, G., Liang, W., Hada, M., Ehara, M., Toyota, K., Fukuda, R., Hasegawa, J., Ishida, M., Nakajima, T., Honda, Y., Kitao, O., Nakai, H., Vreven, T., Throssell, K., Montgomery, J. A., Jr., Peralta, J. E., Ogliaro, F., Bearpark, M. J., Heyd, J. J., Brothers, E. N., Kudin, K. N., Staroverov, V. N., Keith, T. A., Kobayashi, R., Normand, J., Raghavachari, K., Rendell, A. P., Burant, J. C., Iyengar, S. S., Tomasi, J., Cossi, M., Millam, J. M., Klene, M., Adamo, C., 
Cammi, R., Ochterski, J. W., Martin, R. L., Morokuma, K., Farkas, O. ve Foresman, J. B., (2009). Gaussian 09, Revision D.01. Wallingford, CT, Gaussian Inc.

Hadjoudis, E, Mavridis, I. M., Dziembowska, T., Rozwadowski, Z. ve Anulewicz, R., (1999). Spectroscopic and Structural Studies of the Thermochromism of Solid Di-Anil of 2-Hydroxy-5-MethylIsophthaldehyde. Molecular Engineering, 8, 97-104.

Hadjoudis, Eugene ve Mavridis, I. M., (2004). Photochromism and thermochromism of Schiff bases in the solid state: structural aspects. Chemical Society reviews, 33(9), 579-588.

Karabiyik, H., Petek, H., Iskeleli, N. O. ve Albayrak, Ç., (2009). Structural and aromatic aspects for tautomerism of (Z)-6-((4-bromophenylamino)methylene)-2,3-dihydroxycyclohexa-2,4-dienone. Structural Chemistry, 20(6), 1055-1065.

Kaştaş, G. ve Kaştaş Ç. A., (2019). Scrutinizing the two new o-hydroxy Schiff bases from the point of tautomeric behavior and non-covalent interactions (H-bond, $\mathrm{Br} \cdots \mathrm{Br}, \pi \cdots \pi$ and $\mathrm{C}-\mathrm{H} \cdots \pi$ ) in their supramolecular architectures. Journal of Molecular Structure, 1184, 427-434.

Kaur, G., Singh, S., Sreekumar, A. ve Choudhury, A. R., (2016). The evaluation of the role of C-H*FF hydrogen bonds in crystal altering the packing modes in the presence of strong hydrogen bond. Journal of Molecular Structure, 1106, 154-169.

Ledoux, I. ve Zyss, J., (1982). Influence of the molecular environment in solution measurements of the Secondorder optical susceptibility for urea and derivatives. Chemical Physics, 73(1-2), 203-213.

Mercier, G. M., Robeyns, K. ve Leyssens, T., (2016). Altering the Photochromic Properties of NSalicylideneanilines Using a Co-Crystal Engineering Approach. Crystal Growth \& Design, 16(6), 31983205.

O’boyle, N. M., Tenderholt, A. L. ve Langner, K. M., (2008). cclib: A library for package-independent computational chemistry algorithms. Journal of Computational Chemistry, 29(5), 839-845.

Özdemir Tarı, G., Ceylan, Ü., Ağar, E. ve Eserci, H., (2016). Crystal structure, spectroscopic investigations and quantum chemical computational study of 5-(diethylamino)-2-((3-nitrophenylimino)methyl)phenol. Journal of Molecular Structure, 1126, 83-93.

Özdemir Tarı, G., Ceylan, Ü., Uzun, S., Ağar, E. ve Büyükgüngör, O., (2018). Synthesis, spectroscopic (FTIR, UV-Vis), experimental (X-Ray) and theoretical (HF/DFT) study of: (E)-2-Chloro-N-((4nitrocyclopenta-1,3-dienyl)methylene)benzenamine. Journal of Molecular Structure, 1174, 18-24.

Özek Yıldırım, A., Albayrak Kaştaş, Ç. ve Gülsu, M., (2016). Synthesis, structural characterization and computational studies of (E)-4-bromo-2-((3-chlorophenylimino)methyl)-6-ethoxyphenol. Journal of Molecular Structure, 1103, 311-318.

Sarı, H., (2020). Bölüm 11: Doğrusal Olmayan Optik. 10 Ekim 2020 Tarihinde, https://acikders.tuba.gov.tr/pluginfile.php/1070/mod_resource/content/1/Bolum-11.pdf adresinden erişildi

Schiff, H., (1864). Mittheilungen aus dem Universitätslaboratorium in Pisa: eine neue reihe organischer Basen. Justus Liebigs Annalen der Chemie, 131(1), 118-119.

Sengar, M. ve Narula, A. K., (2019). Luminescence Sensitization of Eu(III) Complexes with Aromatic Schiff Base and N,N'-Donor Heterocyclic Ligands: Synthesis, Luminescent Properties and Energy Transfer. Journal of Fluorescence, 29(1), 111-120.

Tezer, N. ve Karakus, N., (2009). Theoretical study on the ground state intramolecular proton transfer (IPT) and solvation effect in two Schiff bases formed by 2-aminopyridine with 2-hydroxy-1-naphthaldehyde and 2-hydroxy salicylaldehyde. Journal of Molecular Modeling, 15(3), 223-232.

Thanthiriwatte, K. S. ve Nalin de Silva, K., (2002). Non-linear optical properties of novel fluorenyl derivatives - ab initio quantum chemical calculations. Journal of Molecular Structure: THEOCHEM, 617(1-3), 169-175.

Tidwell, T. T., (2008). Hugo (Ugo) Schiff, Schiff Bases, and a Century of $\beta$-Lactam Synthesis. Angewandte Chemie International Edition, 47(6), 1016-1020.

Ünver, H., Kabak, M., Zengin, D. M. ve Durlu, T. N., (2001). Keto - enol tautomerism , conformations, and structure. Journal of Chemical Crystallography, 31(4), 203-209.

Ünver, H., Yıldız, M., Kiraz, A. ve Özgen, Ö., (2008). Spectroscopic Studies and Crystal Structure of (Z)-6[(2-hydroxyphenylamino)methylene]-2-methoxycyclohexa-2,4-dienone. Journal of Chemical Crystallography, 39(1), 17-23.

Weigend, F. ve Ahlrichs, R., (2005). Balanced basis sets of split valence, triple zeta valence and quadruple zeta valence quality for $\mathrm{H}$ to Rn: Design and assessment of accuracy. Physical Chemistry Chemical Physics, 7(18), 3297.

Zaitsev, K. V., Piskun, Y. A., Oprunenko, Y. F., Karlov, S. S., Zaitseva, G. S., Vasilenko, I. V., Churakov, 
A.V., ve Kostjuk, S. V., (2014). Controlled ring-opening homo- and copolymerization of $\varepsilon$-caprolactone and D,L-lactide by iminophenolate aluminum complexes: An efficient approach toward well-defined macromonomers. Journal of Polymer Science Part A: Polymer Chemistry, 52(9), 1237-1250.

Zhao, Y. ve Truhlar, D. G., (2008). The M06 suite of density functionals for main group thermochemistry, thermochemical kinetics, noncovalent interactions, excited states, and transition elements: Two new functionals and systematic testing of four M06-class functionals and 12 other function. Theoretical Chemistry Accounts, 120(1-3), 215-241. 